Running Head: TELEOLOGICAL LEARNING

Forthcoming in Journal of Cognition and Development

\title{
Young children's learning and generalization of teleological and mechanistic explanations
}

\author{
Tania Lombrozo ${ }^{1}$ \\ Elizabeth Baraff Bonawitz ${ }^{2}$ \\ Nicole R. Scalise ${ }^{3}$ \\ ${ }^{1}$ Department of Psychology, University of California, Berkeley \\ ${ }^{2}$ Department of Psychology, Rutgers University - Newark \\ ${ }^{3}$ Department of Human Development and Quantitative Methodology, University of \\ Maryland, College Park
}

\section{Author Note}

Tania Lombrozo, Department of Psychology, University of California at Berkeley;

Elizabeth Baraff Bonawitz, Department of Psychology, Rutgers University - Newark; Nicole R.

Scalise, Department of Human Development and Quantitative Methodology, University of Maryland, College Park.

Correspondence concerning this article should be addressed to Tania Lombrozo, Department of Psychology, University of California at Berkeley, 3210 Tolman Hall, Berkeley, CA, 94720. E-mail: lombrozo@berkeley.edu.

\section{Acknowledgments}

We thank the members of the Concepts and Cognition Lab and the Gopnik Lab at UC Berkeley for helpful feedback and support and to Si Wang for help with preliminary coding. The reported experiment was completed in partial fulfillment of the undergraduate thesis requirements for N. Brooke, and was partially funded by NSF grant BCS-0819231 to T. Lombrozo and a Jacobs Foundation Fellowship to L. Bonawitz. 


\begin{abstract}
Young children often endorse explanations of the natural world that appeal to functions or purpose, explaining (for example) that rocks are pointy so animals can scratch on them. By contrast, most Western-educated adults reject such explanations. What accounts for this change? We investigated 4- to 5-year-old children's ability to generalize the form of an explanation from examples by presenting them with novel teleological explanations, novel mechanistic explanations, or with no explanations for five nonliving natural objects. They were then asked to explain novel instances of the same objects and novel kinds of objects. We found that children were able to learn and to generalize explanations of both types, suggesting an ability to draw generalizations over the form of an explanation. We also found that teleological and mechanistic explanations were learned and generalized equally well, suggesting that if a domain-general teleological bias exists, it does not manifest as a bias in learning or generalization.
\end{abstract}

Keywords: teleological explanation, functional explanation, mechanistic explanation, explanatory preferences, learning, generalization 
Young children's learning and generalization of teleological and mechanistic explanations

The popular children's book Look Look! endearingly concludes by telling its young readers that "stars shine all for you" (Linenthal, 1998). While Western adults might balk at this purposive take on properties of the nonliving natural world, it isn't foreign to most young children. In fact, given the choice between explaining why particular rocks are pointy by appeal to a purpose ("so that animals...could scratch on them") or by appeal to a mechanical process ("because little bits of stuff piled up on top of one another over a long time"), most elementary school students opt for explanations like the former (Kelemen, 1999a; see also Piaget, 1929; Sully, 1900). With age and education, however, this tendency becomes more selective (e.g., Casler \& Kelemen, 2008; Kelemen, 1999b). For most Western adults, "teleological" or "functional" explanations - explanations that appeal to a purpose, function, or goal - are restricted to artifacts and some biological traits (Lombrozo \& Carey, 2006). So while most will accept that streetlights shine "for us," they won't say the same about stars.

Why do children's teleological preferences change over the course of development? Past work convincingly shows that culture and education play important roles. For example, by 6 years of age, secular Israeli children show a weaker and more circumscribed preference for teleological explanations than orthodox Israeli children (Diesendruck \& Haber, 2009; see also Casler \& Kelemen, 2008; Kelemen, 2003; Rottman et al., 2017). There's also evidence that adults with greater exposure to western education are more selective in their application of teleological explanations (Casler \& Kelemen, 2008; Sánchez Tapia et al., 2016). Moreover, culture can affect the content of teleological explanations: a study comparing Quechua-speaking Peruvians with Americans found that the former group 
produced more teleological explanations involving ecological relationships (Sánchez Tapia et al., 2016; see also ojalheto, Waxman, \& Medin, 2013). These findings indicate that there must be some mechanism(s) by which exposure to particular cultural or pedagogical materials, practices, or contexts affects the perceived scope of teleological explanations.

One possibility is that as children hear particular explanations, they form generalizations concerning the kind of explanation involved (i.e., teleological or mechanistic) and the kind of entity to which it is being applied (e.g., a human artifact, or a nonliving natural thing). For example, after hearing many mechanistic explanations for nonliving natural things (e.g., those mountains resulted from volcanic activity, the rain is a result of water droplets forming in clouds...), children could form the generalization that nonliving natural things tend to support mechanistic explanations. But the prerequisites to this kind of generalization are not trivial: children must be able to represent kinds of explanations as such and to group objects into classes that have some correspondence to explanation type. In fact, the single study that attempted to teach children to produce mechanistic explanations did not succeed: Kelemen (1999a, 2003) attempted to train children to select mechanistic explanations over teleological alternatives by providing them with an example of how a scientist would explain cloud formation (e.g., clouds "are all made up of tiny drops of water and sometimes when the water drops get really cold then it rains," p. 207, Kelemen, 2003). This training had very little effect on subsequent explanation choices, with most children endorsing teleological explanations for most item types.

In the current study, we aim to investigate whether children can successfully generalize an explanation type from one case to another by implementing a training regime 
that might be a better match to children's everyday experience. Rather than providing a single extended example with the additional instructions to answer "like a scientist," we present children with multiple explanations of a particular type, and we then assess learning and generalization. Specifically, we introduce 4- and 5-year-old children to five examples of nonliving natural objects (e.g., stars) from a fictional planet, Bizorm. Each object is introduced with no explanation (e.g., "Wow, look at this!"), with a teleological explanation ("Stars on Bizorm are very bright yellow so that people can see them"), or with a mechanistic explanation ("Gas burns in the stars on Bizorm so they are very bright yellow"). We then solicit explanations from children in two tasks: a learning test and a transfer test. In the learning test, we assess how effectively the provided explanations were remembered and applied by asking children to explain novel instances of the same kinds of objects (e.g., another star). In the transfer test, we ask children to explain the properties of nonliving objects or phenomena that were not previously seen (e.g., a river).

The transfer test is especially crucial for assessing whether the type of explanation modeled in training generalizes to novel items. To do so, we can see whether the children in the teleological training condition generate more teleological explanations on the transfer test than do children in the control condition (who receive no training), and whether the children in the mechanistic training condition generate more mechanistic explanations on the transfer test than do children in the control condition. In both cases it's important to consider children's responses relative to the control condition, rather than in absolute terms, to ensure that preferences for one explanation type over the other are driven by the training itself, and not by the spontaneous explanatory preferences that children might have concerning those items, even in the absence of training. 
By testing children's learning and transfer for both mechanistic and teleological explanations, our experiment also has the potential to shed light on ongoing debates about the scope of teleological reasoning. According to one perspective, teleology reflects a "default" and domain-general preference to reason about the world in terms of function and purpose (Kelemen, 1999c). This perspective is supported by evidence that children accept teleological explanations across domains (Kelemen, 1999a), and that this preference is not a simple consequence of parental input: parents do not provide a disproportionate number of teleological explanations in child-directed speech (Kelemen, Callanan, Casler, Pérez-Granados, 2005). Moreover, adults err in the direction of accepting scientificallyunwarranted teleological explanations when responding under time pressure (Kelemen \& Rosset, 2009; Kelemen, Rottman, \& Seston, 2013) or when cognitively impaired (Lombrozo, Kelemen, \& Zatchik, 2007), suggesting that a preference for teleological explanations could persist as a default preference throughout the lifespan.

An alternative perspective is that teleological explanations reflect a more selective "design stance" that is restricted to reasoning about the products of intentional or apparent design, namely artifacts and biological adaptations (Keil, 1992, 1994). Supporting this view, Greif, Kemler Nelson, Keil, and Gutierrez (2006) found that children sometimes asked function-seeking questions for artifacts (e.g., "what is it for?"), but never did so for animals. It's also worth noting that while children do tend to accept teleological explanations for animals and for non-living natural things when they are offered, they do so significantly more often for artifacts and for biological adaptations, suggesting some selectivity operating over what may be a baseline preference. Similarly, while adults accept some scientifically-unwarranted teleological explanations under speeded conditions (e.g. 
"earthworms tunnel underground to aerate the soil"), they do not accept teleological explanations that are treated as "bad" control items (e.g., "cars have horns to illuminate dark roads") (Kelemen \& Rosset, 2009). These findings suggest some selectivity in the scope of teleological explanations.

Given these ongoing debates, a secondary goal of the present research is to revisit questions about the selectivity of teleological explanations using our novel method. Instead of focusing on which explanations children produce (e.g., Sánchez Tapia et al., 2016) or select (e.g., Kelemen, 1999), or on the function-seeking questions children ask (Greif et al., 2006), we focus on how readily children learn and generalize novel explanations when they are offered. If children favor teleological explanations as a cognitive default, we might expect this to manifest as a learning bias, with teleological explanations more readily learned and generalized than mechanistic explanations, provided such explanations are not already at ceiling. In contrast, if children show some early selectivity in their use of teleological explanations, we might instead expect mechanistic explanations to be more readily learned and generalized in our task, given that the domain of non-living natural objects does not typically involve actual or apparent design. Our method thus provides a new way to address long-standing questions about the selectivity of young children's teleological preferences.

In sum, our experiment investigates how young children learn and generalize teleological and mechanistic explanations by presenting them with no explanations, with novel teleological explanations, or with novel mechanistic explanations, and then soliciting explanations for matched and novel cases. Our study is among the first to consider whether children can form generalizations over explanation types, effectively extending a mode of 
explanation from trained instances to novel cases. Our study can also inform ongoing debates about the scope of young children's teleological preference.

\section{Methods}

Participants. Sixty children (mean: 54 months; range: 47-71) were recruited from local preschools $(N=58)$ or a science museum $(N=2)$ and randomly assigned to one of three conditions: neutral baseline, teleological training, or mechanistic training. There were 20 children in each condition, with no significant differences in age, $F(2,57)=1.362, p=$ .264. One additional child was replaced due to experimenter error. The children represented a broad range of ethnicities.

Materials. A picture book was used in training and included five drawings of nonliving natural objects (pond, mountain, cave, island, star). The learning test for this picture book included an additional five drawings of these objects, modified to be similar but distinguishable. The transfer task used drawings of five new natural objects or phenomena (desert, canyon, thunder, river, volcano). Sample illustrations are provided in Figure 1. (The complete set of stimuli can be found in online supplemental materials.)

\section{Procedure}

Training task. Children were introduced to the five-page training book with drawings of nonliving natural objects from the planet "Bizorm" (see Figure 1, top). A statement accompanied each drawing (see Table 1). In the Neutral baseline condition the experimenter provided a neutral statement (e.g., "Wow, look at this!"). In the Teleological training condition the experimenter provided a teleological explanation (e.g., "Caves on Bizorm are very dark so that animals can hide in them."). In the Mechanistic training 
condition the experimenter provided a mechanistic explanation (e.g., "There are no holes for light to shine through in caves on Bizorm, so they are very dark.").

Learning test. After observing all training items, children received the learning test (see Figure 1, middle). The original drawings remained visible as the experimenter introduced five new drawings (e.g., "Here is another cave from the planet Bizorm”). Children were then asked why each property held for the objects in the new drawings (e.g., “Why do you think this cave is so dark?"). Children were first prompted to provide a free response. After the free response, they were presented with a forced response option that included the explanations provided during the teleological and mechanistic training conditions, with order counterbalanced.

Transfer test. Children were shown five drawings of nonliving natural objects or phenomena from planet Earth (see Figure 1, bottom). As with the learning test, children were prompted to explain a property of each item and then given a forced-choice option between teleological and mechanistic explanations, with order counterbalanced (see Table 2).

\section{Explanation Coding And Preliminary Analyses}

Free response coding. Free-response explanations were coded into three nonoverlapping categories: "teleological" (appealing to a function or purpose, e.g., "the cave is dark so animals can sleep and hide there from hunters"); "mechanistic" (appealing to proximate causal processes, e.g., "the light can't shine because the holes [are] so small"); or "other" (e.g., repetition of the property in question or description of the picture, "that's how caves are," "this one's big and this one's small”). Eighty percent of children's responses 
were coded by both the first and third authors, blind to condition; kappa was .70. The analyses reported below correspond to those of the first coder, who coded all responses.

It's worth noting that for the learning test in the teleological and mechanistic training conditions, participants could in principle have produced a teleological or mechanistic explanation that did not match the one offered in training. However, this happened very rarely - about seven times in the teleological training condition and twice in the mechanistic training condition (less than $6 \%$ of total explanations). This helps rule out the concern that the teleological and mechanistic explanations that we happened to use might be differentially memorable. Moreover, the explanations that were produced in these cases were often related to the explanations originally provided. For example, one child trained that caves are dark so that animals can hide in them offered the free response that they are dark "so they can sleep in there," and a child taught that stars are bright so that people can see them explained that stars are bright "so people can see in the dark." To allow for masked coding and consistency in coding across conditions, we therefore coded explanations as "teleological" or "mechanistic" irrespective of the child's condition or whether the explanation matched the one that was offered in training.

Forced choice coding. For the forced choice responses, we summed the number of teleological explanations chosen for the learning and transfer tests. Overall, participants chose teleological explanations on 2.57 of the 5 learning trials $(S D=1.49)$, and on 2.49 of the of the 5 transfer trials $(S D=1.02)$, neither of which was significantly different from chance, $t(59)=.347, p=.730$, and $t(59)=-.381, p=.704$ (one-sample t-tests). Moreover, forced-choice responses did not differ as a function of condition, even for learning items, $F(2,57)=1.718, p=.189$. The forced choice response was given to all children, even those 
who had provided a free response that matched one of the options provided; however, this follow-up likely prompted children to change their answer (Gonzalez et al, 2012; Bonawitz et al, in review). Given this methodological concern and children's chance responding on this secondary measure, we restrict analyses to the original free response data.

\section{Results}

We were interested in two main questions pertaining to children's learning of the explanations and their generalizations. First, how readily did children learn the particular teleological or mechanistic explanations provided during training (as reflected by the learning test), and did children learn one type of explanation more readily than the other? Second, how readily did children generalize the modeled explanation type to novel kinds of objects (as reflected by the transfer test), and did children generalize one type of explanation more readily than the other? We address these questions in turn.

To address the first question, we analyzed free responses on the learning task as a function of condition (see Figure 2). Children produced an average of $1.22(S D=1.777)$ teleological explanations (from a possible range of $0-5$ ), and this number varied as a function of condition, $F(2,57)=45.567, p<.001, \eta_{p}^{2}=.615$. Children produced $3.20(S D=$ 1.795) teleological explanations in the teleological condition, $.30(S D=.657)$ in the neutral condition, and $.15(S D=.489)$ in the mechanistic condition. Post-hoc Tukey tests revealed that responses in the teleological condition differed significantly from the other two conditions, $p<.001$, which did not differ from each other, $p=.909$.

Because the distribution of teleological responses was skewed towards zero in the mechanistic and neutral conditions, we also conducted a non-parametric independentsamples Kruskal-Wallis test, which confirmed that the distribution of responses differed as 
a function of condition, $p<.001$. Comparing pairs of conditions with an independentsamples Mann-Whitney U test similarly confirmed that the teleological condition differed significantly from the mechanistic and neutral conditions, $p s<.001$, which did not differ from each other, $p=.602$.

For mechanistic explanations, children produced an average of 1.30 such responses $(S D=1.660)$ (from a possible range of $0-5)$ on the learning test, and this number also varied as a function of condition, $F(2,57)=34.635, p<.001, \eta_{p}{ }^{2}=.549$. Children produced $.20(S D=.410)$ mechanistic explanations in the teleological condition, $.70(S D=1.174)$ in the neutral condition, and $3.00(S D=1.522)$ in the mechanistic condition. Post-hoc Tukey tests revealed that responses in the mechanistic condition differed significantly from the other two conditions, $p<.001$, which did not differ from each other, $p=.351$.

Once again, the distribution of mechanistic responses was skewed towards zero in the teleological and neutral conditions. We thus conducted a non-parametric independentsamples Kruskal-Wallis test, which confirmed that the distribution of responses differed as a function of condition, $p<.001$. Comparing pairs of conditions with an independentsamples Mann-Whitney U test similarly confirmed that the mechanistic condition differed significantly from the teleological and neutral conditions, $p s<.001$, which did not differ from each other, $p=.314$.

We next considered whether teleological and mechanistic explanations were learned differentially. To do so, we first coded responses in the teleological and mechanistic conditions as training-consistent, i.e., teleological explanations produced in the teleological training condition were coded as matches, whereas mechanistic explanations produced in the mechanistic training condition were coded as matches. Simply comparing the 
proportion of matches across conditions, however, could reflect differences in the test items related to their bias for teleological versus mechanistic explanations, and not differential effects of learning as a function of the training. We therefore treated responses in the neutral condition as an indication of how "teleologically-biased" or "mechanisticallybiased" the items were, and used these responses as a baseline correction for the teleological and mechanistic training conditions. Specifically, we subtracted the mean number of teleological explanations produced on learning items in the neutral condition (i.e., .30) from each teleological match score in the teleological condition, and the mean number of mechanistic explanation produced in the neutral condition (i.e., .70) from each mechanistic match score in the mechanistic condition.

Having computed these baseline-corrected match scores, which reflect the extent to which a training condition increased the rate of training-consistent responses over the (neutral condition) baseline, we used an independent-samples $t$-test to compare the rate of training-consistent responses produced in the teleological versus mechanistic conditions. The result was not significant (teleological: $M=2.900, S D=1.795$; mechanistic: $M=2.300$, $S D=1.522), t(38)=-1.140, p=.261$. Addressing our first question, then, these results suggest that children were able to effectively learn some of the explanations provided during training, but that teleological and mechanistic explanations were learned equally readily.

To address our second question, we analyzed free responses on the transfer task as a function of condition (see Figure 3). Children produced an average of .62 (SD=1.106) teleological explanations (from a possible range of 0-5), and this number varied as a function of condition, but only marginally, $F(2,57)=2.459, p=.095, \eta_{p}^{2}=.079$. Children 
produced $1.05(S D=1.395)$ teleological explanations in the teleological condition, $.35(S D=$ $.489)$ in the neutral condition, and $.45(S D=1.146)$ in the mechanistic condition. Post-hoc Tukey tests revealed that no two conditions differed significantly, $p s>.01$. Because responses in all conditions were skewed towards zero, we also conducted a nonparametric independent-samples Kruskal-Wallis test, which similarly failed to find a significant effect of condition, $p=.126$.

For mechanistic explanations, children produced an average of $2.60(S D=1.392)$ such responses (from a possible range of 0-5), and this number varied as a function of condition, $F(2,57)=6.333, p=.003, \eta_{p}{ }^{2}=.182$. Children produced $2.00(S D=1.214)$ mechanistic explanations in the teleological condition, $2.40(S D=1.392)$ in the neutral condition, and $3.40(S D=1.231)$ in the mechanistic condition. Post-hoc Tukey tests revealed that responses in the mechanistic condition differed significantly from the other two conditions, $p<.05$, which did not differ from each other, $p=.588$. While responses were normally distributed, we followed up with non-parametric tests, which mirrored these patterns of significance.

To evaluate whether teleological and mechanistic explanations were differentially generalized, we again coded the number of training-consistent responses produced in the teleological and mechanistic training conditions, and subtracted the mean number of responses of the corresponding type from the neutral condition to serve as a baseline correction. An independent-samples $t$-test comparing these difference scores was not significant (teleological: $M=.700, S D=1.395$; mechanistic: $M=1.000, S D=1.231), t(38)=$ $.721, p=.475$. Addressing our second question, then, these results suggest that children 
were able to generalize the explanation type learned during training, but that teleological and mechanistic explanations were generalized equally readily.

\section{General Discussion}

Our findings suggest that when it comes to nonliving natural objects such as stars and caves, 4- to 5-year-old children are able to learn novel teleological and mechanistic explanations, and to generalize these kinds of explanation to novel items. Moreover, they are able to learn and generalize these two explanation types equally well. Below we consider possible interpretations and implications of our results.

Our primary research question concerned the mechanism(s) by which children's explanatory preferences change over the course of development. Given that culture and education are both factors, it's plausible that children are able to generalize from individual explanations that they encounter, coming to learn which kinds of explanations apply to particular kinds of cases. Our findings suggest that children are indeed able to generalize in this way, with several examples of a particular explanation type sufficient for them to generate completely novel explanations of that same type. We speculate that our training was successful, while the training from Kelemen (2003) was not, because we provided children with multiple examples rather than a single case. From these examples, they were able to extract the common explanatory form despite variation in content, suggesting some representation of explanation type with respect to which they could note similarities.

Our findings do not reveal the precise nature of the generalization that children drew. Past work suggests that different kinds of explanations have different causal commitments (Lombrozo \& Carey, 2006). In particular, children are more likely to accept teleological explanations when they believe the entity being explained was designed or 
made by some creator (Kelemen \& DiYanni, 2005). One possibility is that children who received teleological explanations inferred that the objects on planet Bizorm are created or designed, and it's this causal commitment that was generalized to novel objects. Another possibility is that explanatory generalizations were made over the explanatory form itself, without concomitant commitments about causal etiology. This is an important question for future research.

Our secondary research questions concerned whether teleological and mechanistic explanations might be learned or generalized differentially. We did not find such effects. Children were able to learn and to generalize both types of explanations for nonliving, natural kinds, with no significant differences between them. This result potentially speaks against the idea of a teleological bias. Insofar as such a bias exists, it did not manifest on our task. Similarly, our results run counter to the idea that children favor mechanistic explanations in the domain of nonliving natural things; while there was a baseline preference (at least in the generalization test items), there was not a bias in learning or generalization. That said, these conclusions follow from a null result, and should thus be interpreted with caution. Research on cultural evolution suggests that even very small biases can generate large differences over time (e.g., Kalish, Griffiths, \& Lewandowsky, 2007) and influence the nature of culturally accepted beliefs (Boyer, 2001; Boyer \& Ramble, 2001). So even if children start out with a very weak bias favoring one type of explanation over the other, this bias could manifest as a strong preference later in development or later in the course of cultural evolution.

Our study has several limitations. Given the known effects of age, culture, and education on explanatory preferences, findings from a single sample should be generalized 
with caution. Our study was also restricted to the domain of nonliving natural things. Future work could investigate a wider range of domains, and in so doing explore children's ability to draw generalizations not only over kinds of explanations, but also over appropriate kinds of entities.

In sum, we find that children are able to learn and to generalize novel teleological and mechanistic explanations, and to do so equally well for both types of explanations. So while young children may occasionally hear that stars "shine all for you," our findings suggest that a greater preponderance of mechanistic explanations in their environment could be enough to curb their teleological tendencies towards the natural world as they grow older. 


\section{References}

Bonawitz, E.B., Shafto, P., Yu, Y., Bridgers, S., \& Gonzalez, A. (in review) Children rationally change their beliefs in response to neutral follow-up questions

Bonawitz, E.B. \& Lombrozo, T. (2012). Occam's Rattle: Children's use of simplicity and probability to constrain inference. Developmental Psychology, 48(4), 1156-1164.

Boyer, P. (2001). Religion Explained: The Evolutionary Foundations of Religious Belief. Basic Books.

Boyer, P. \& Ramble, C. (2001). Cognitive templates for religious concepts: cross-cultural evidence for recall of counter-intuitive representations. Cognitive Science, 25, 535-564.

Casler, K. \& Kelemen, D. (2008). Developmental continuity in teleo-functional explanation: Reasoning about nature among Romanian Romani adults. Journal of Cognition and Development, 9(3), 340-362.

Diesendruck, G. \& Haber, L. (2009). God's categories: The effect of religiosity on children's teleological and essentialist beliefs about categories. Cognition, 110, 100-114.

Gonzalez, A., Shafto, P., Bonawitz, E., \& Gopnik, A. (2012) Is that your final answer? The effects of neutral queries on children's choices. In Miyake, N., Peebles, D., \& Cooper, R. (Eds.) Proceedings of the Thirty-fourth Cognitive Science Society, 1614-1619.

Greif, M., Kemler-Nelson, D., Keil, F.C. and Gutierrez, F. (2006). What do children want to know about animals and artifacts?: Domain-specific requests for information. Psychological Science, 17(6), 455-459.

Kalish, M.L., Griffiths, T.L., \& Lewandowsky, S. (2007). Iterated learning: intergenerational knowledge transmission reveals inductive biases. Psychonomic Bulletin and Review, 14, 288-294. 
Keil, F.C. (1992). The origins of an autonomous biology. In M.R. Gunnar \& M. Maratsos (Eds.), The Minnesota symposia on child psychology: Vol. 25. Modularity and constraints in language and cognition (pp. 103-137). Hillsdale, NJ: Erlbaum.

Keil, F.C. (1994). The birth and nurturance of concepts by domains: The origins of concepts of living things. In L.A. Hirschfeld \& S.A. Gelman (Eds.), Mapping the mind: Domain specificity in cognition and culture (pp. 234-254). New York: Cambridge University Press.

Keil, F. (2006). Explanation and understanding. Annual Review of Psychology, 57, 227-254.

Kelemen, D. (1999a). Why are rocks pointy?: Children's preference for teleological explanations of the natural world. Developmental Psychology, 35, 1440-1453.

Kelemen, D. (1999b). Functions, goals and intentions: Children's teleological reasoning about objects. Trends in Cognitive Sciences, 12, 461-468.

Kelemen, D. (1999c). The scope of teleological thinking in preschool children. Cognition, 70, $241-272$.

Kelemen, D. (1999d). Beliefs about purpose: On the origins of teleological thought. In M. and S. Lea (Eds.). The Descent of Mind. Oxford: Oxford University Press.

Kelemen, D. (2003). British and American children's preferences for teleological-functional explanations of the natural world. Cognition, 88, 201-221.

Kelemen, D., Callanan, M., Casler, K. \& Pérez-Granados, D. R. (2005). Why things happen: Teleological explanation in parent-child conversations. Developmental Psychology, 41(1), 251-264.

Kelemen, D., \& DiYanni, C. (2005). Intuitions about origins: Purpose and intelligent design in children's reasoning about nature. Journal of Cognition and Development, 6(1), 3-31. 
Kelemen, D. \& Rosset, E. (2009). The Human Function Compunction: Teleological explanation in adults. Cognition, 111, 138-143.

Kelemen, D., Rottman, J., \& Seston, R. (2013). Professional physical scientists display tenacious teleological tendencies: Purpose-based reasoning as a cognitive default. Journal of Experimental Psychology: General, 142(4), 1074.

Linenthal, P. (1998). Look Look! New York, NY: Dutton Children's Books.

Lombrozo, T. (2006). The structure and function of explanations. Trends in Cognitive Sciences, 10, 464-470.

Lombrozo, T. (2012). Explanation and abductive inference. K.J. Holyoak and R.G. Morrison (Eds.), Oxford Handbook of Thinking and Reasoning (pp. 260-276), Oxford, UK: Oxford University Press.

Lombrozo, T. \& Carey, S. (2006). Functional explanation and the function of explanation. Cognition, 99(2), 167-204.

Lombrozo, T., Kelemen, D. \& Zaitchik, D. (2007). Inferring design: Evidence of a preference for teleological explanations in patients with Alzheimer's disease. Psychological Science, 18(11), 999-1006.

ojalheto, B. Waxman, S. R., \& Medin, D. L. (2013). Teleological reasoning about nature: intentional design or relational perspectives?. Trends in cognitive sciences, 17(4), 166171.

Piaget, J. (1929). The child's conception of the world. London: Routledge \& Kegan Paul.

Rottman, J., Zhu, L., Wang, W., Seston Schillaci, R., Clark, K. J., \& Kelemen, D. (2017). Cultural influences on the teleological stance: evidence from China. Religion, Brain \& Behavior, 7(1), 17-26. 
Sánchez Tapia, I., Gelman, S. A., Hollander, M. A., Manczak, E. M., Mannheim, B., \& Escalante, C. (2016). Development of Teleological Explanations in Peruvian QuechuaSpeaking and US English-Speaking Preschoolers and Adults. Child development, 87(3), $747-758$.

Sully, J. (1900). Studies of Childhood. New York, NY: D. Appleton \& Company. 
Table 1. Statements that accompanied each of the five items in the training task for each condition, along with the corresponding question that was later asked on the learning test.

\begin{tabular}{|c|c|c|c|c|}
\hline Item & $\begin{array}{c}\text { Neutral } \\
\text { Baseline }\end{array}$ & $\begin{array}{c}\text { Teleological } \\
\text { Training }\end{array}$ & $\begin{array}{c}\text { Mechanistic } \\
\text { Training }\end{array}$ & Test question \\
\hline Pond & $\begin{array}{l}\text { "Wow, look at } \\
\text { this!" }\end{array}$ & $\begin{array}{l}\text { "Ponds on } \\
\text { Bizorm are very } \\
\text { still so that the } \\
\text { water doesn't } \\
\text { spill out" }\end{array}$ & $\begin{array}{l}\text { "No rocks fall } \\
\text { into ponds on } \\
\text { Bizorm, so they } \\
\text { are very still" }\end{array}$ & $\begin{array}{l}\text { "Why do you } \\
\text { think this pond } \\
\text { is so still?" }\end{array}$ \\
\hline Cave & $\begin{array}{l}\text { "Wow, look at } \\
\text { this!" }\end{array}$ & $\begin{array}{l}\text { "Caves on } \\
\text { Bizorm are very } \\
\text { dark so that } \\
\text { animals can } \\
\text { hide in them" }\end{array}$ & $\begin{array}{l}\text { "There are no } \\
\text { holes for light to } \\
\text { shine through in } \\
\text { caves on } \\
\text { Bizorm, so they } \\
\text { are very dark" }\end{array}$ & $\begin{array}{l}\text { "Why do you } \\
\text { think this cave } \\
\text { is so dark?" }\end{array}$ \\
\hline Star & $\begin{array}{l}\text { "Wow, look at } \\
\text { this!" }\end{array}$ & $\begin{array}{l}\text { "Stars on } \\
\text { Bizorm are very } \\
\text { bright yellow so } \\
\text { that people can } \\
\text { see them" }\end{array}$ & $\begin{array}{l}\text { "Gas burns in } \\
\text { the stars on } \\
\text { Bizorm, so they } \\
\text { are very bright } \\
\text { yellow" }\end{array}$ & $\begin{array}{l}\text { "Why do you } \\
\text { think this star is } \\
\text { so bright } \\
\text { yellow?" }\end{array}$ \\
\hline Mountain & $\begin{array}{l}\text { "Wow, look at } \\
\text { this!" }\end{array}$ & $\begin{array}{l}\text { "Mountains on } \\
\text { Bizorm are very } \\
\text { pointy so that } \\
\text { animals don't } \\
\text { climb on them" }\end{array}$ & $\begin{array}{l}\text { "Mountains on } \\
\text { Bizorm are very } \\
\text { pointy because } \\
\text { smooth pieces } \\
\text { of mountain fall } \\
\text { off" }\end{array}$ & $\begin{array}{l}\text { "Why do you } \\
\text { think this } \\
\text { mountain is so } \\
\text { pointy?" }\end{array}$ \\
\hline Island & $\begin{array}{l}\text { "Wow, look at } \\
\text { this!" }\end{array}$ & $\begin{array}{l}\text { "Islands on } \\
\text { Bizorm are very } \\
\text { small so that } \\
\text { ships don't } \\
\text { bump into } \\
\text { them" }\end{array}$ & $\begin{array}{l}\text { "Islands on Bizorm } \\
\text { are very small } \\
\text { because the ocean } \\
\text { covers most of the } \\
\text { land" }\end{array}$ & $\begin{array}{l}\text { Why do you } \\
\text { think this island } \\
\text { is so small?" }\end{array}$ \\
\hline
\end{tabular}


Table 2. Complete set of items for the transfer test, including the teleological and mechanistic explanations provided for the forced choice question.

\begin{tabular}{|c|c|c|c|}
\hline Item & Question & Teleological & Mechanistic \\
\hline Desert & $\begin{array}{l}\text { Why do you think } \\
\text { this desert is so hot? }\end{array}$ & $\begin{array}{l}\text { [Or] do you think it's } \\
\text { so that people don't } \\
\text { walk on it? }\end{array}$ & $\begin{array}{l}\text { [Or] do you think it's } \\
\text { because there are no } \\
\text { trees to shade it? }\end{array}$ \\
\hline River & $\begin{array}{l}\text { Why do you think } \\
\text { this river is so } \\
\text { narrow? }\end{array}$ & $\begin{array}{l}\text { [Or] do you think it's } \\
\text { so that animals can } \\
\text { cross over it? }\end{array}$ & $\begin{array}{l}\text { [Or] do you think it's } \\
\text { because not very } \\
\text { much water goes } \\
\text { through it? }\end{array}$ \\
\hline Thunder & $\begin{array}{l}\text { Why do you think } \\
\text { this thunder is so } \\
\text { loud? }\end{array}$ & $\begin{array}{l}\text { [Or] do you think it's } \\
\text { so that people know } \\
\text { to go inside? }\end{array}$ & $\begin{array}{l}\text { [Or] do you think it's } \\
\text { because lightening in } \\
\text { the clouds makes a } \\
\text { noise? }\end{array}$ \\
\hline Canyon & $\begin{array}{l}\text { Why do you think } \\
\text { this canyon is so } \\
\text { deep? }\end{array}$ & $\begin{array}{l}\text { [Or] do you think it's } \\
\text { so that things on this } \\
\text { side can't cross over } \\
\text { to the other side? }\end{array}$ & $\begin{array}{l}\text { [Or] do you think it's } \\
\text { because little pieces } \\
\text { fell away over a long } \\
\text { time? }\end{array}$ \\
\hline Volcano & $\begin{array}{l}\text { Why do you think } \\
\text { this volcano has } \\
\text { steam on top? }\end{array}$ & $\begin{array}{l}\text { [Or] do you think it's } \\
\text { so that people know } \\
\text { to stay away from it? }\end{array}$ & $\begin{array}{l}\text { [Or] do you think it's } \\
\text { because the hot lava } \\
\text { heated up the inside } \\
\text { of the volcano? }\end{array}$ \\
\hline
\end{tabular}


Figure 1. Sample stimuli from the picture books used in training (top; pointy mountain and bright stars), in the learning test (middle; pointy mountain and bright stars), and in the transfer test (bottom; hot desert and steaming volcano).

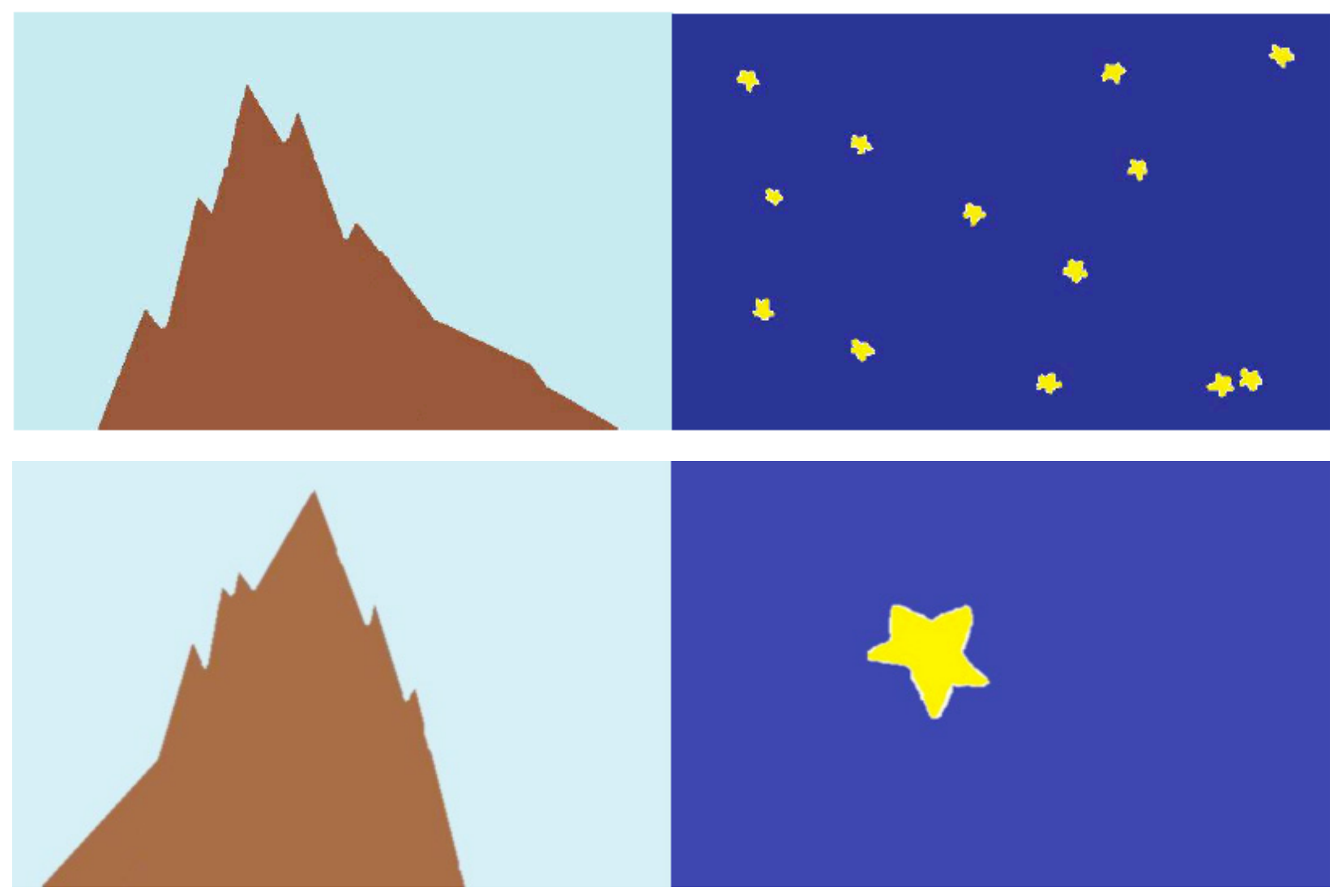


Figure 2. The mean number of teleological and mechanistic explanations offered (of 5) on the learning test as a function of training condition. Error bars correspond to one SEM in each direction.

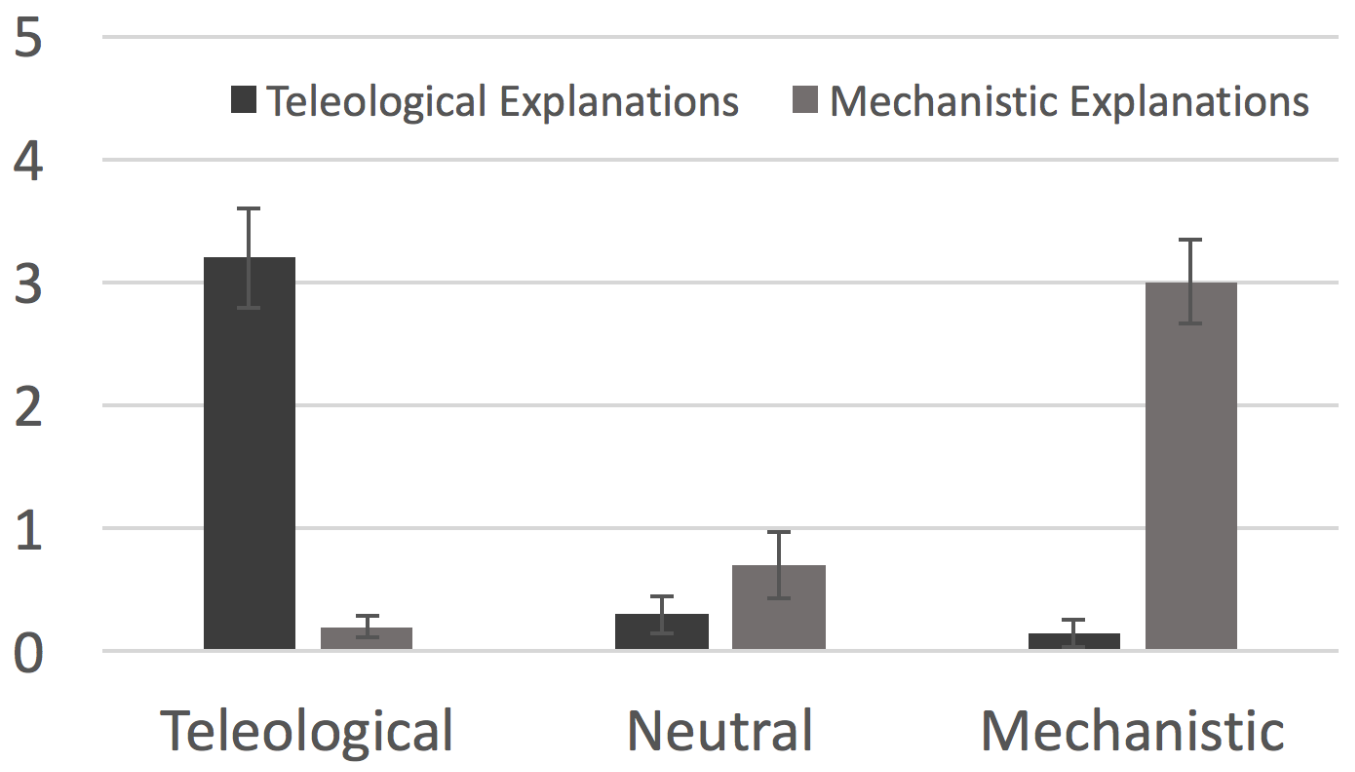


Figure 3. The mean number of teleological and mechanistic explanations offered (of 5) on the transfer test as a function of training condition. Error bars correspond to one SEM in each direction.

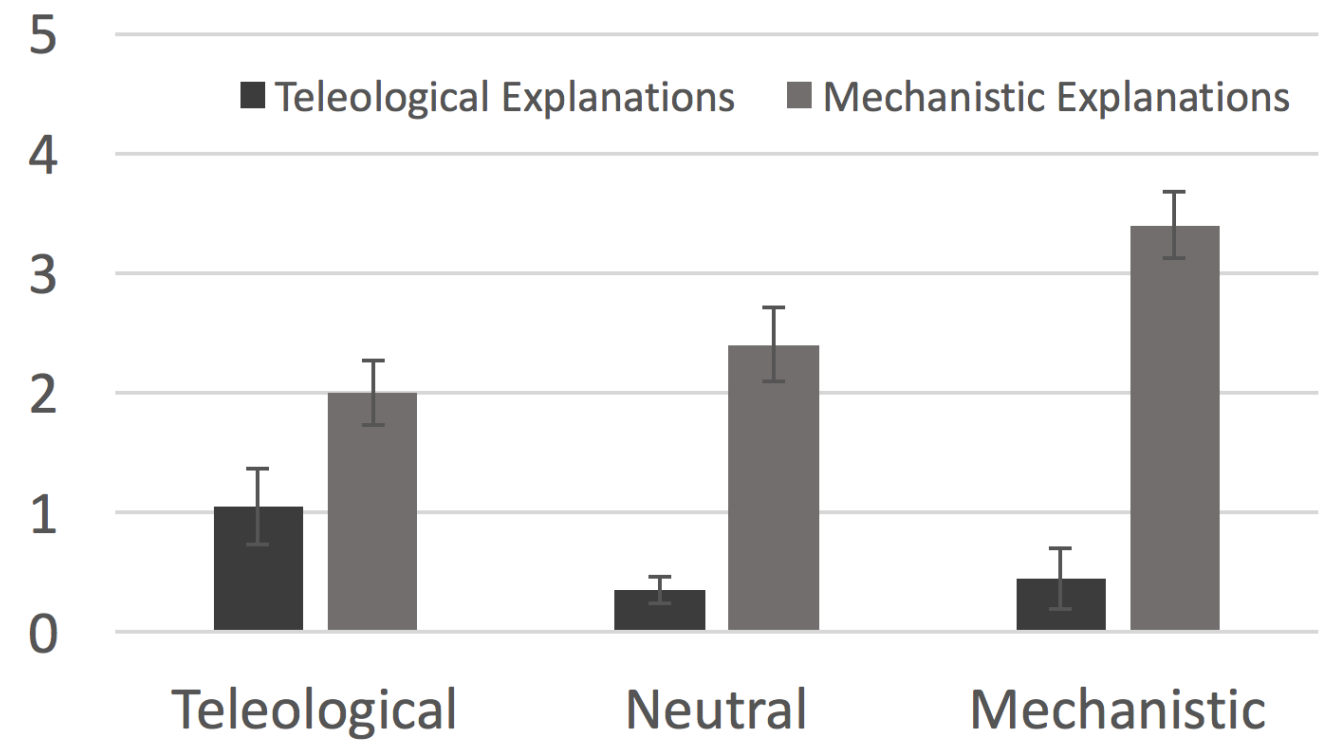




\section{Supplemental Materials}

\section{A. The five picture books used in training task (original and test).}

\section{Pond}

Original

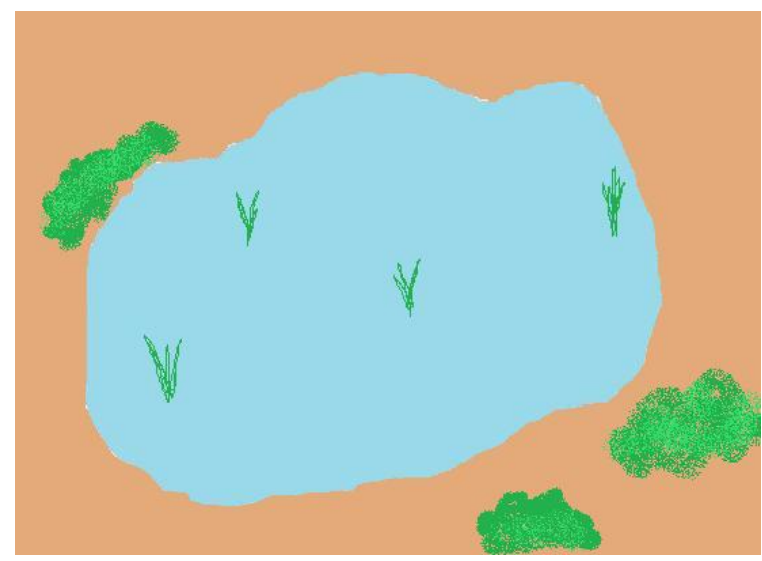

Mechanistic training: No rocks fall into ponds on Bizorm, so they are very still.

Teleological training: Ponds on Bizorm are very still so that the water doesn't spill out.

Neutral statement: Wow, look at this!

\section{Mountain}

\section{Original}

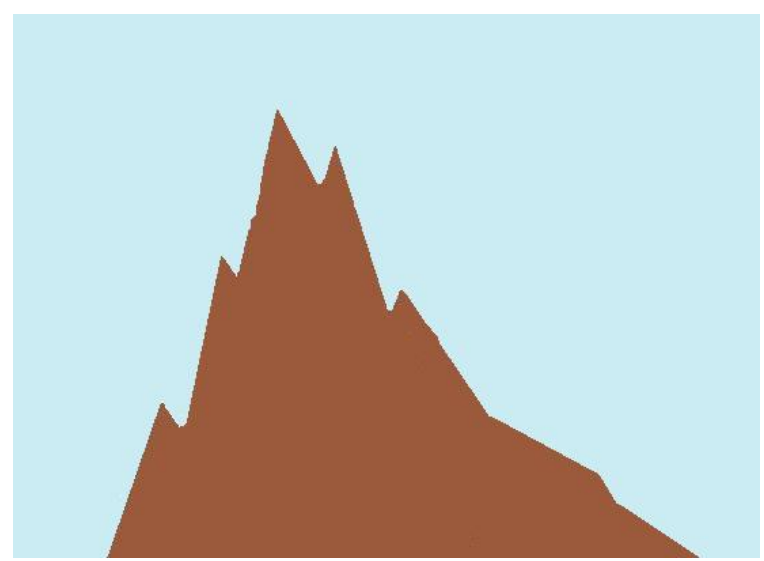

Mechanistic training: Mountains on Bizorm are very pointy because smooth pieces of mountain fall off.

Teleological training: Mountains on Bizorm are very pointy so that animals don't climb on
Test

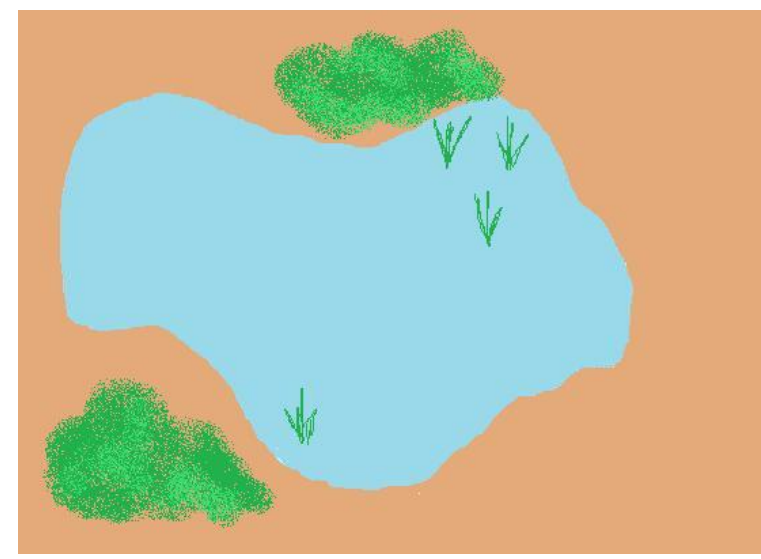

Here is another pond from the planet Bizorm. Why do you think this pond is so still?

Test

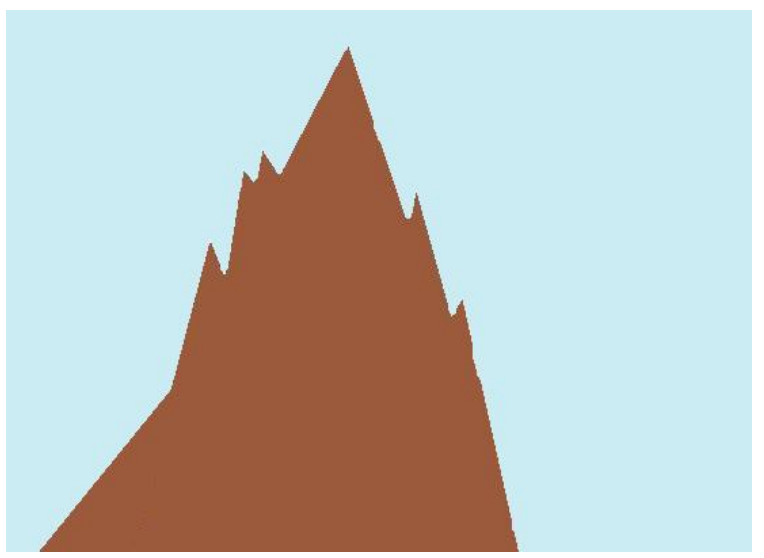

Here is another mountain from the planet Bizorm. Why do you think this mountain is so pointy? 
them.

Neutral statement: Wow, look at this!

\section{Cave}

Original

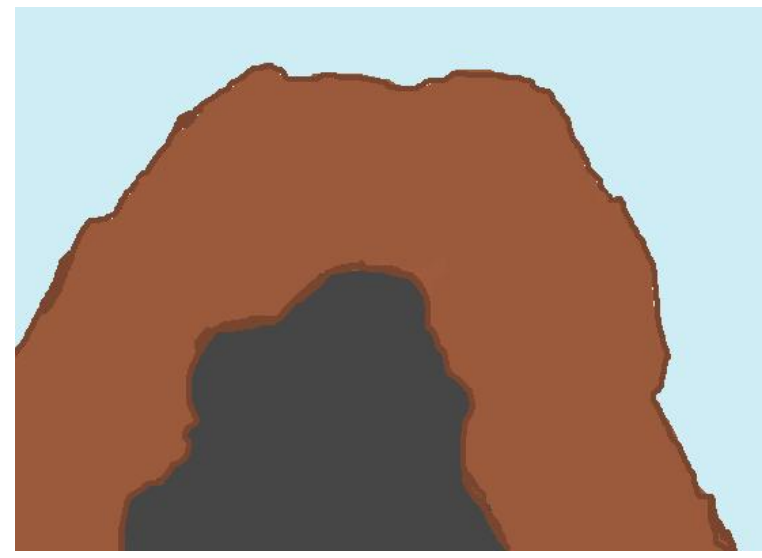

Mechanistic training: There are no holes for light to shine through in caves on Bizorm, so they are very dark.

Teleological training: Caves on Bizorm are very dark so that animals can hide in them Neutral statement: Wow, look at this!

\section{Island}

\section{Original}

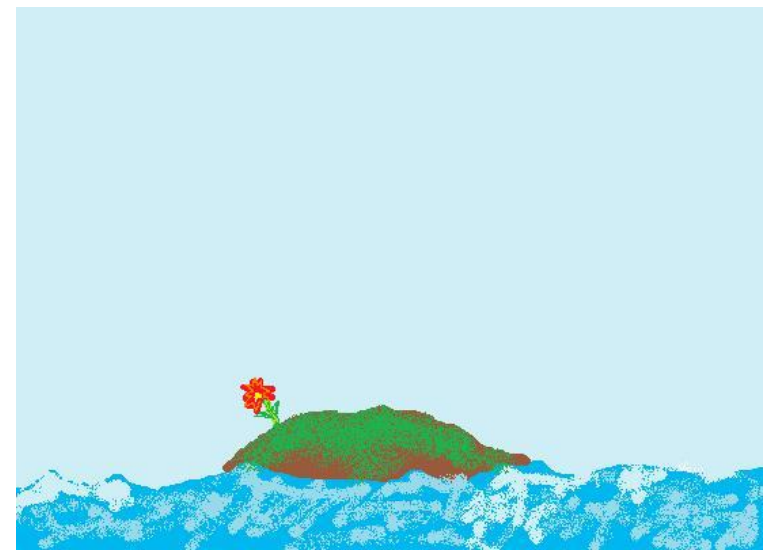

Mechanistic training: Islands on Bizorm are very small because the ocean covers most of the land.

Teleological training: Islands on Bizorm are very small so that ships don't bump into them.
Test

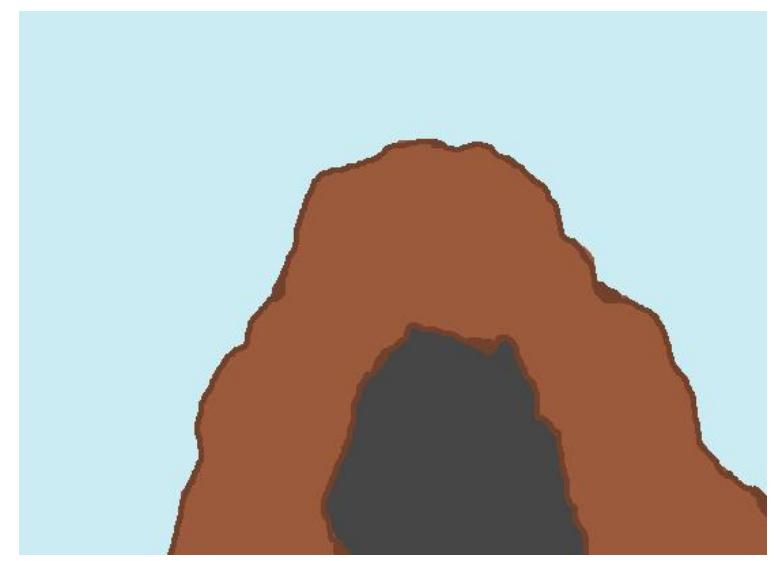

Here is another cave from the planet Bizorm. Why do you think this cave is so dark?

Test

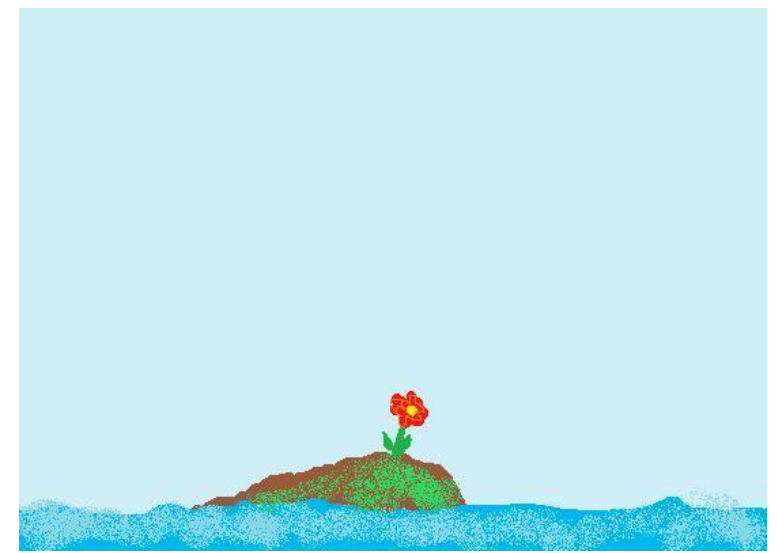

Here is another island from the planet Bizorm. Why do you think this island is so small? 
Neutral statement: Wow, look at this!

Star

Original

Test
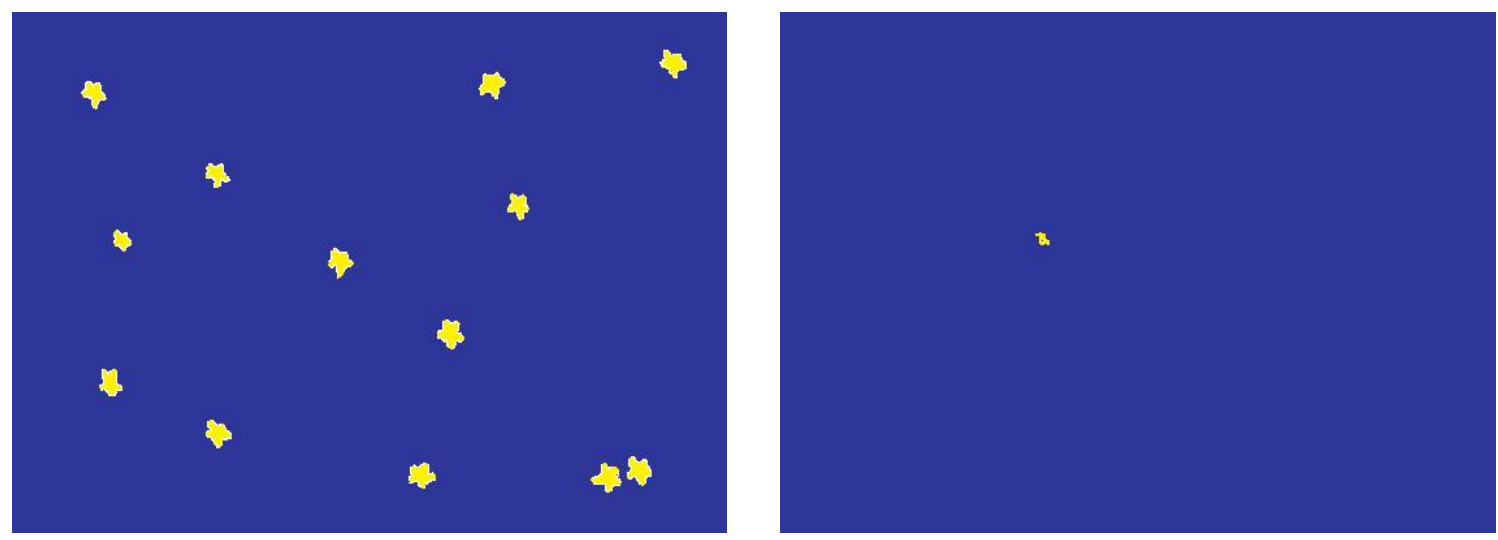

Mechanistic training: Gas burns in the stars on Bizorm, so they are very bright yellow.

Here is another star from the planet Bizorm. Why do you think this star is so bright yellow?

Teleological training: Stars on Bizorm are very bright yellow so that people can see them.

Neutral statement: Wow, look at this!

\section{B. The five picture books used in the transfer task.}

\section{Desert}

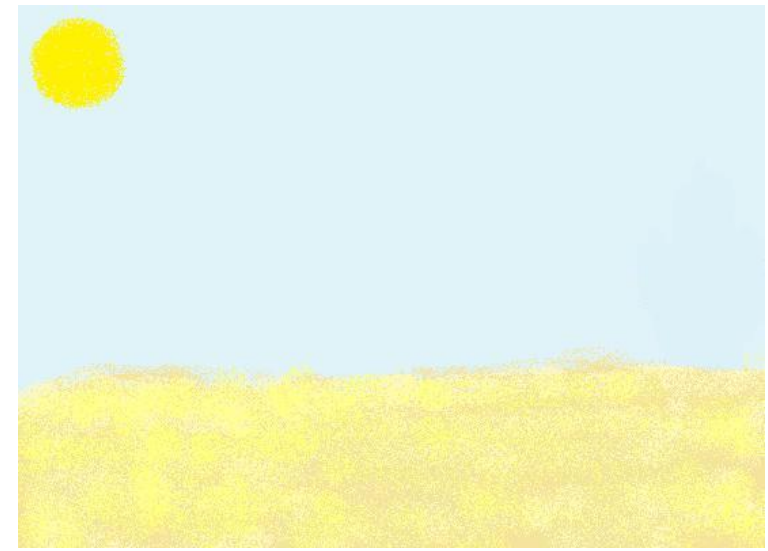

"Why do you think this desert is so hot?"

\section{River}

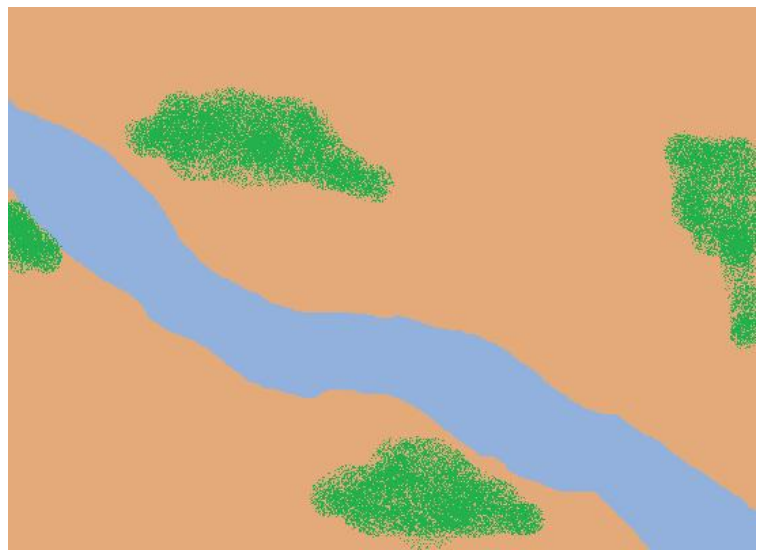

"Why do you think this river is so narrow?" 


\section{Thunder}

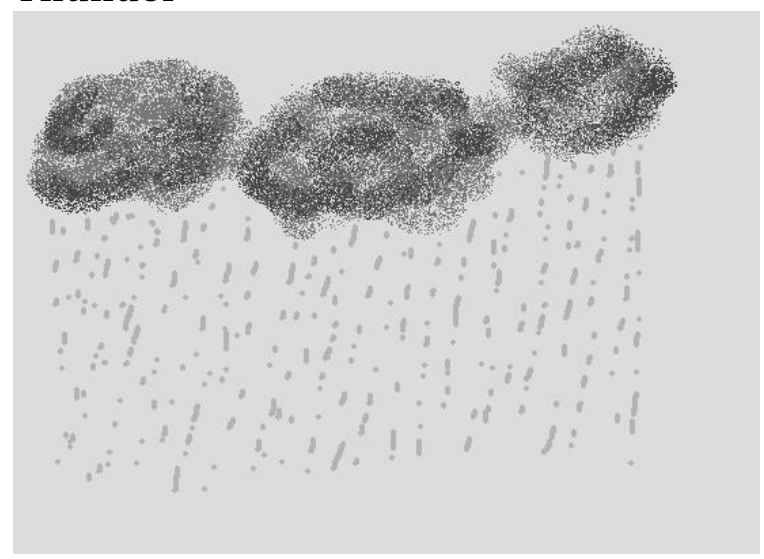

"Why do you think this thunder is so loud?"

\section{Canyon}

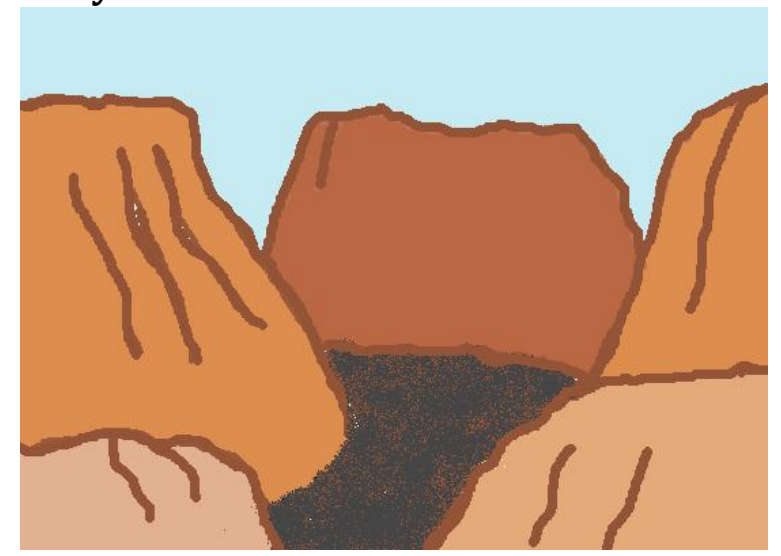

"Why do you think this canyon is so deep?"

\section{Volcano}

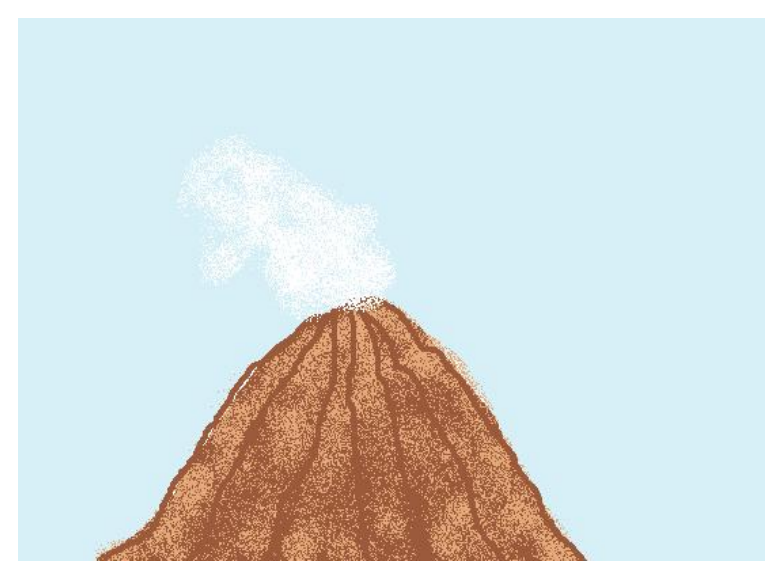

"Why do you think this volcano has steam on top?" 